\title{
Design and Development of a Mobile Rover- OCTAGON
}

\author{
Dr. Tadaga Channaveerappa Manjunath \\ Ph.D. (IIT Bombay), Fellow-IETE, Member-IEEE \\ Director- Research \& Development, Atria Institute of Technology \\ Bangalore, Karnataka, India
}

\begin{abstract}
The paper presents the design and fabrication of each part and construction of a 4 axes mobile robot with indigenous components and to control it with a personal computer using the java language. The main objective of this work is to design, fabricate a modular simple low-cost indigenously developed educational full fledged robotic control system named as "OCTAGON" that could accomplish 2 dimensional motion in a XY plane equipped with intelligence system and a 4 DOF manipulator arm for Pick aNd Place (PNP) operations from the source to the destination provided by the user. The robot is simulated and modeled using the graphic simulators and the AutoCAD simulation package. A user-friendly module developed in JAVA is designed and successfully implemented for the operation of the entire system to do some specific operations [41].
\end{abstract}

\section{General Terms}

Robot, PC, Control System.

\section{Keywords}

Intelligence, Pick \& Place, Obstacles, Mobile robot, JAVA, AutoCAD, Controller, Arm.

\section{INTRODUCTION}

Imagine a day in your life when you wake up in the morning and find a machine walking up to you \& saying 'GOOD MORNING SIR ! HAVE A CUP OF TEA'. How would you respond to such a situation? With so much progress made in the field of science, engineering and technology, this dream is absolutely realizable in this automation age. People think that in the near future robots are going to take the place of humans and slowly mankind will come to extinction. But, this is absolutely a false notion.

Robots are being built only to help the mankind. They can work in hazardous areas; can do the jobs, which are beyond our capabilities. Human force will always be required to maintain and manage these robots. Cars are made without humans, surgeries are performed with precision. Most manufacturing units in advanced nations use robots in almost each and every field of science and engineering. No place has been left untouched. The deepest oceans have been explored with the robots and not even space is too greater challenge for the robots and with the extensive usage of robots in the nuclear zones for detection of radiation. Besides robots do have some amount of limitations and also can't do some of the things, which humans can do very easily.

After having gone through all these vast advantages of robots, here, we try to build a prototype of an industrial robot which can do simple pick and place operations of objects which is moving on a smooth surface such as a floor. In the present work, we try to incorporate some artificial intelligence features of the robotic system in the software and developing a user-friendly graphical user interface in JAVA to control all the operations that are taking place in the robotic system. This work had its origin in our own fascination for the field of robotics. That, coupled with our genuine urge to build something substantial, led us to undertaking this endeavour. The task, however, was colossal. Since robotics encompasses everything from mechanics to electronics to computer software, we had the opportunity to learn more than we could have otherwise. Nevertheless, we are proud of our efforts and, more so, of the end result. Presenting the "OCTAGON" as shown in Fig. 1.

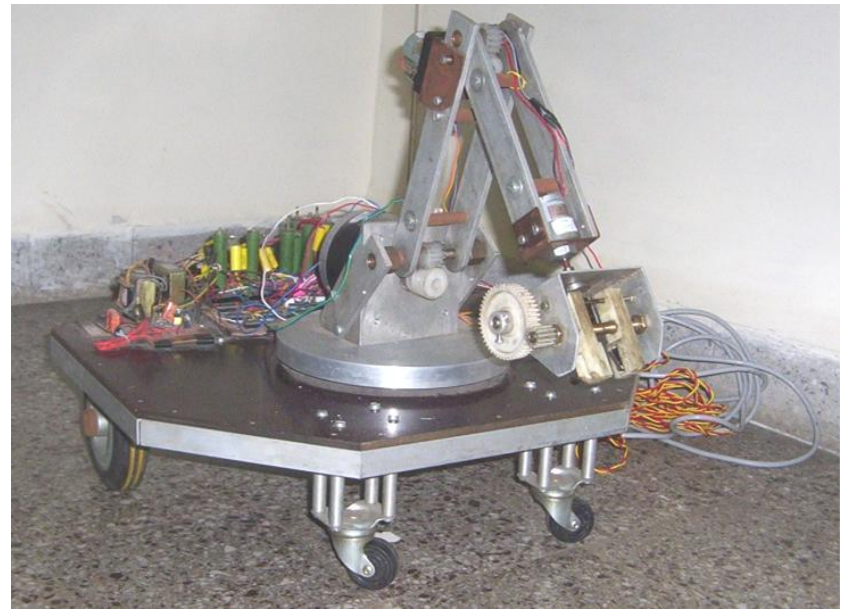

Fig. 1 : The designed OCTAGON

A comprehensive study of the designed, fabricated unit is done by dividing into 3 separate parts as ....

- The mechanical assembly - The mechanical design consist of a mobile trolley, a base, a 2 DOF arm, and the wrist, which has a roll axis and a parallel jaw as the end-effecter.

- The electronic subsystem - The electronic subsystem consist of power supply, relay bank card, L293D based motor driver circuit, an infrared detection circuit and a parallel port interface to a personal computer.

- The computer software for control - The software is written in JAVA and is user friendly, and incorporates many operating modes.

The paper is organized as follows. Section 2 gives a brief introduction to the design and fabrication of the mechanical structure of the robot. Section 3 introduces the reader to the electronics interface while the next section deals with the design of the graphical user interface using JAVA language. Conclusions are presented at the end followed by the references. 

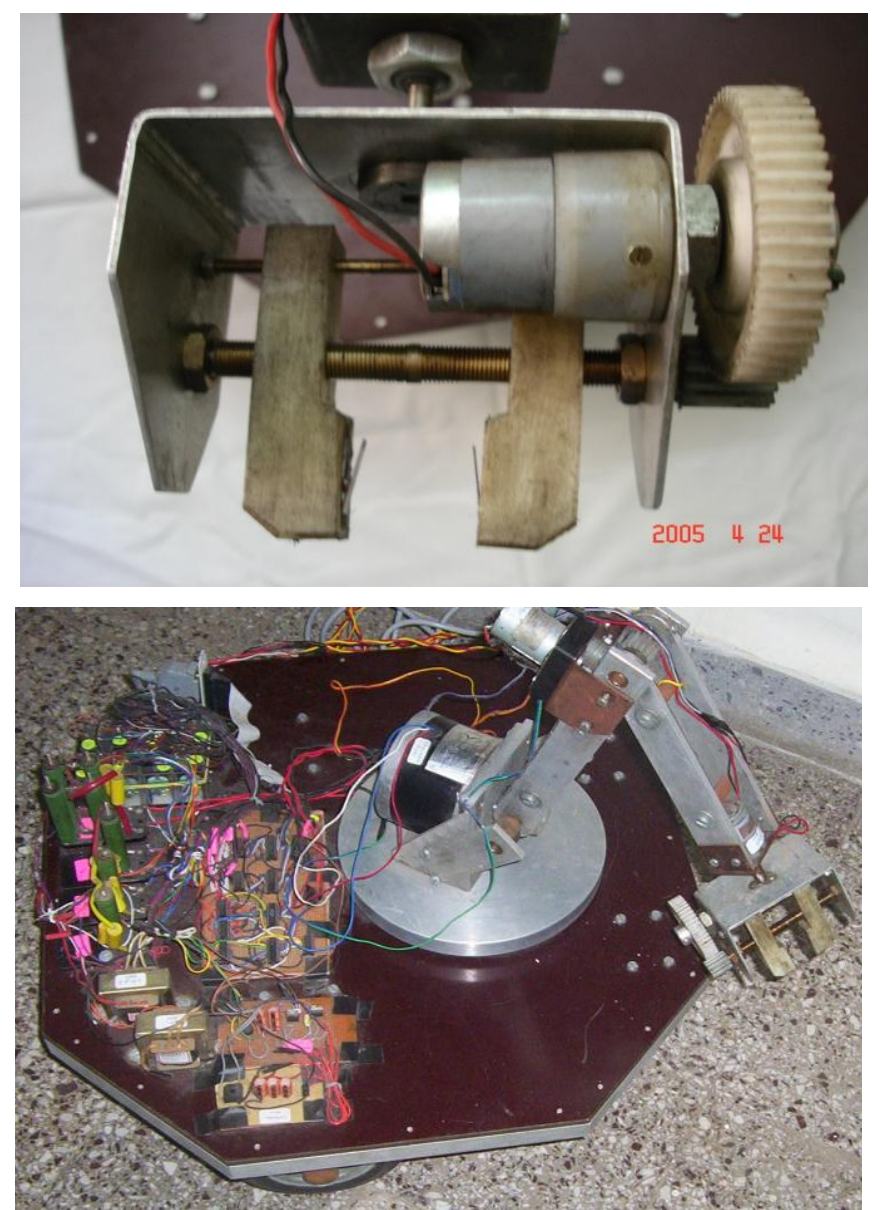

Fig. 2 : Robot with the gripper as the end-effector

\section{DESIGN OF THE MECHANICAL UNIT}

Imagine a day in your life when you wake up in the morning and find a machine walking up to you \& saying 'GOOD MORNING SIR ! HAVE A CUP OF TEA'. How would you respond to such a situation ? With so much progress made in the field of science, engineering and technology, this dream is absolutely realizable in this automation age. People think that in the near future robots are going to take the place of humans and slowly mankind will come to extinction. But, this is absolutely a false notion [41].

The mechanical design of the system is divided into 3 parts, viz., the mobile base assembly (vehicle), the arm assembly and the gripper assembly. The designed robot arm mounted on the vehicle has R-R-R (Rotary-Rotary-Rotary) type of axes and is an articulated one. It has $4 \mathrm{DOF}$ namely, the base rotation, shoulder up / down, elbow up / down, wrist roll [1].

The robot being a PNP-type has a gripper as the end-effector as shown in Fig. 2. The gripper is of parallel jaw type, which works on the principle of left-hand / right-hand screw. These 2 jaws run on the LH / RH screw. The LH / RH screw is made by tapping a brass rod with $\mathrm{LH}$ die from one end and $\mathrm{RH}$ die from other end. The robot moves on 2 main wheels, one at the left and the other at the right with 2 castor wheels, one at the front and other at the back.
This ensures that the gripper jaws move in the opposite direction, that is, the jaws move either towards each other to grip an object or away from each other to release it. The designed and fabricated mobile robotic system consists of a mobile trolley made of hard hylam as shown in the Figs. 1 and 2 over which the entire arm and the necessary interfacing circuitries, electronic and electrical components, accessories are mounted [2].

Bi-directional AC synchronous motors (generating a torque of 10 $\mathrm{kg}-\mathrm{cm}, 1 \mathrm{~A}$ at $60 \mathrm{rpm}$ ) with relay bank switching and DC servos (generating a torque of $5 \mathrm{~kg}-\mathrm{cm}, 0.5 \mathrm{~A}$ current rating, bidirectional, 60 r.p.m) are used to impart motion to the robot. A computer is used for visual display of user program.

\section{DESIGN OF THE ELECTRONIC UNIT}

The mechanical set up forms the skeleton of the robot and what adds intelligence to it is the electronics and the software module. The block diagram of the designed robotic control system is shown in Fig. 5. The electronic design is divided into various sections and finally, the integration is done as shown in Fig. 2 in the photograph [5]. Electronic system consists of various cards like the power supply card, controller card, and driver card, interfacing card, relay bank-switching card and the sensor cards.

The motor drive unit accepts logical inputs from the buffer and accordingly give amplified large output signal to drive the motors. To drive the main AC synchronous motor there is relay drive circuit, which also controls the relay status to achieve desired motion. Computer interfacing of the electronic circuitry through the parallel port is done through a 25 pin male connector, which connects the driver circuit to the PC through the parallel port [3].

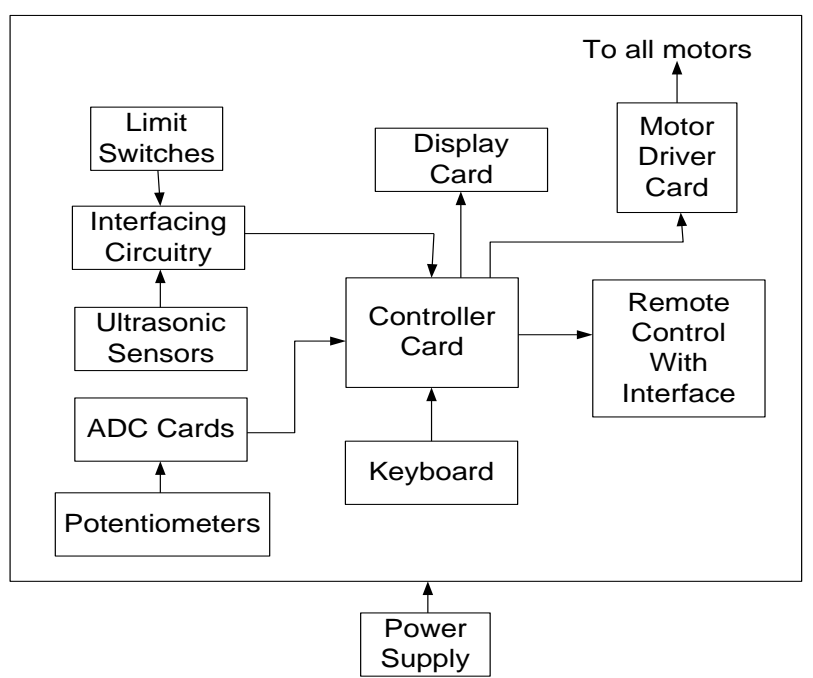

Fig. 3 : Designed \& fabricated interfacing card

The program sends the byte combination to energize the desired motors, which reaches the driver circuit through the 3-meter data cable to the input of the driver circuit. Short circuit prevention logic is also provided as a precautionary measure. Out of the many circuitries used in the electronics, here, the power supply circuitry is shown for convenience. The overall block diagram of the electronics unit along with the power supply unit is shown in the Fig. 4. Various views of electronics are shown in Fig. 5. 


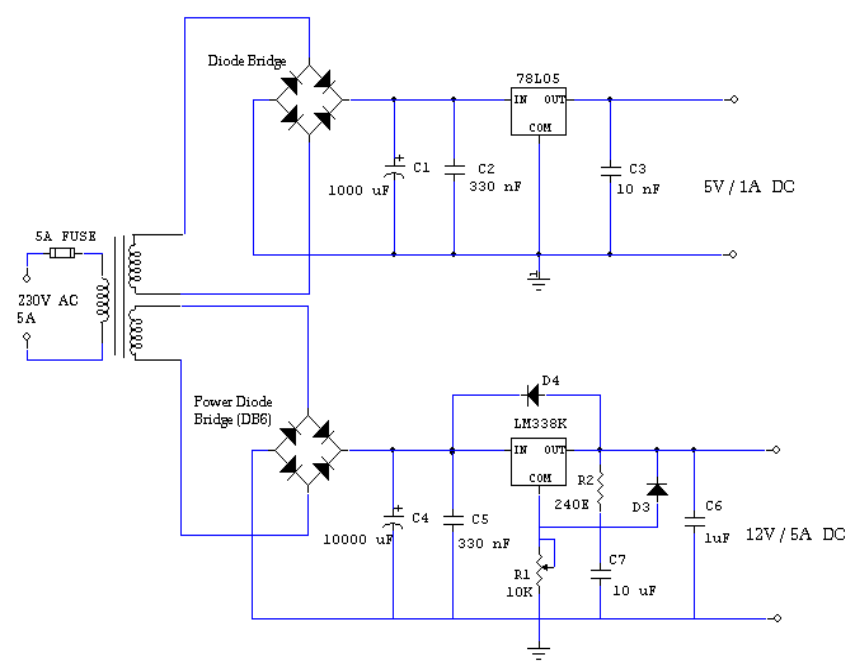

Fig. 4 : Power supply circuitry section

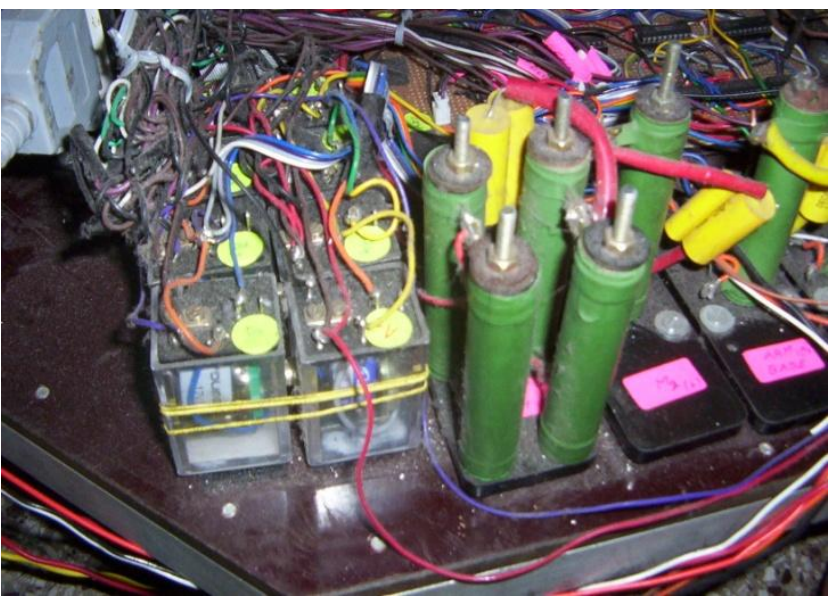

(a)

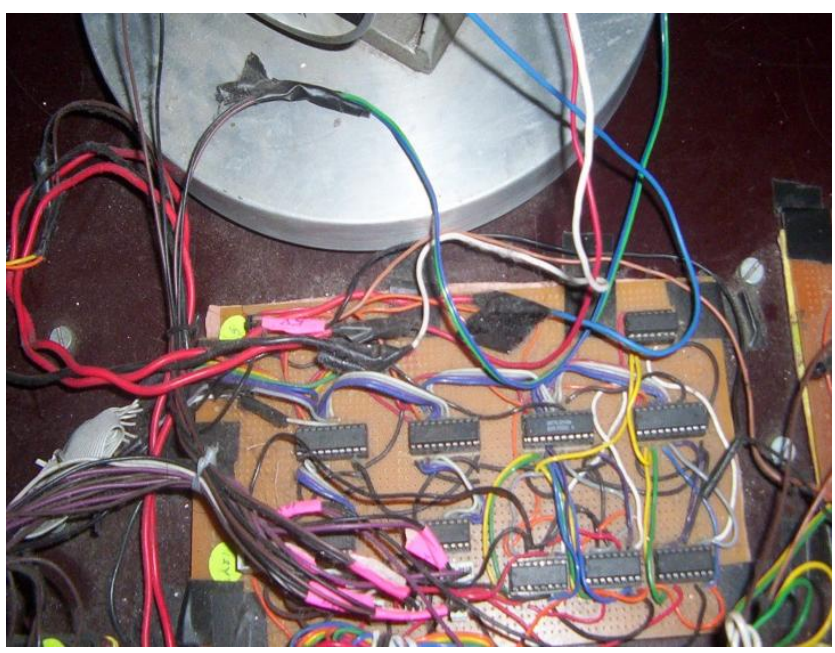

(b)

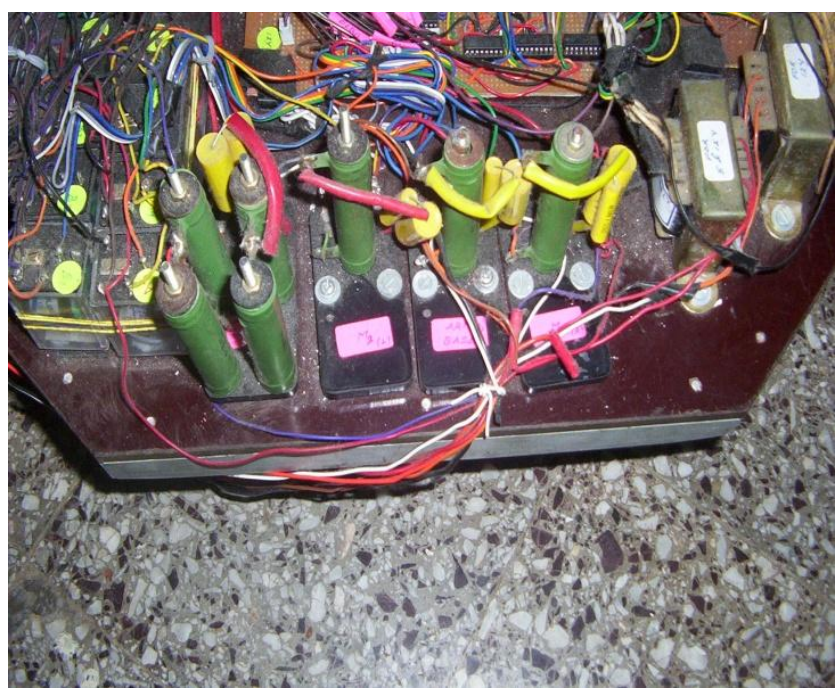

(c)

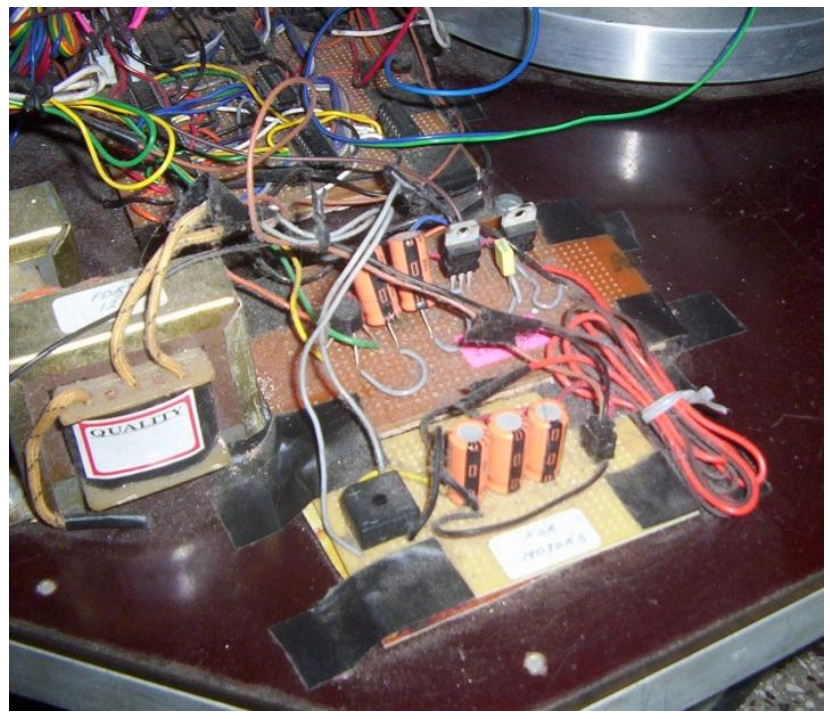

(d)

Fig. 5: Various views of the robot/ electronics

\section{DESIGN OF THE SOFTWARE GUI}

OCTAGON employs a sophisticated application - controlling interface created in JAVA, as it is a fantastic programming language for any application software development. The developed software module flow-chart for controlling the robot is shown in Fig. 9. The software module application facilitates the user interaction with OCTAGON and has many in built features such as the security and authentication.

When the java code is executed, the default GUI screen appears as shown in the Figs. 6 and all the motors status is displayed. For activating a particular motion, we can navigate to its page by selected the left hand menu. The software is designed for maximum robot control \& working efficiency. It is so designed that the user can have complete control over each movable part of the robot [6]. 
Also, the user can easily manoeuvre the robot \& make it traverse a path towards the object to be picked. He or she can then manipulate the different limbs of the robotic arm so that the gripper comes closer to the object \& eventually picks it up. Throughout this process the software interface guides the user through the usage of various parts of the robot \& provides him with responses from the robot.

Given the time on hand, the ease of programming and power of JAVA, it was an ideal choice for use in designing our application interface to the octagon. The software is designed for maximum robot control \& working efficiency. It is so designed that the user can have complete control over each movable part of the robot. Also the user can easily maneuver the robot $\&$ make it traverse a path towards the object to be picked. He can then manipulate the different limbs of the robotic arm so that the gripper comes closer to the object \& eventually picks it up. Throughout this process the software interface guides the user through the usage of various parts of the robot \& provides him with responses from the robot [7].

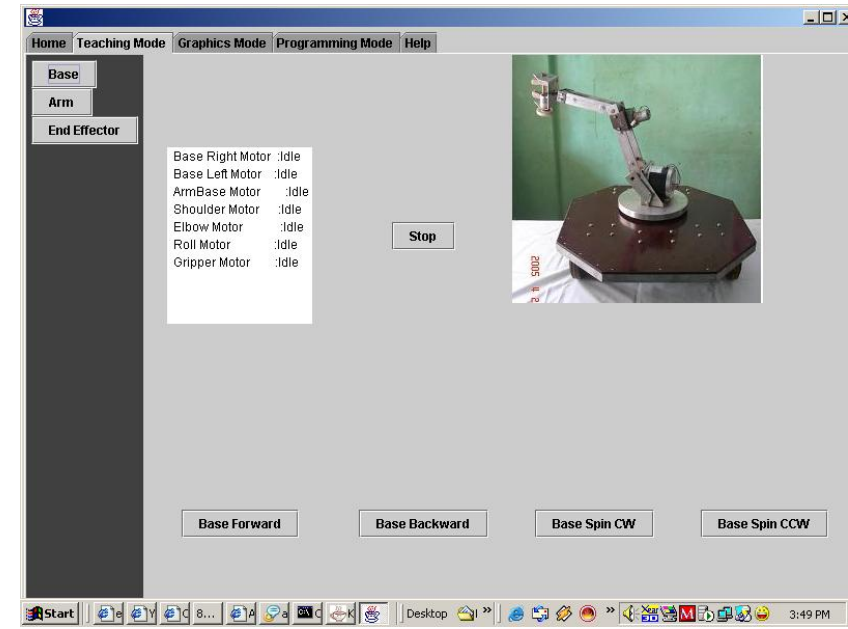

Fig. 6 : General Graphical User Interface (GUI) for the robot control via JAVA environment

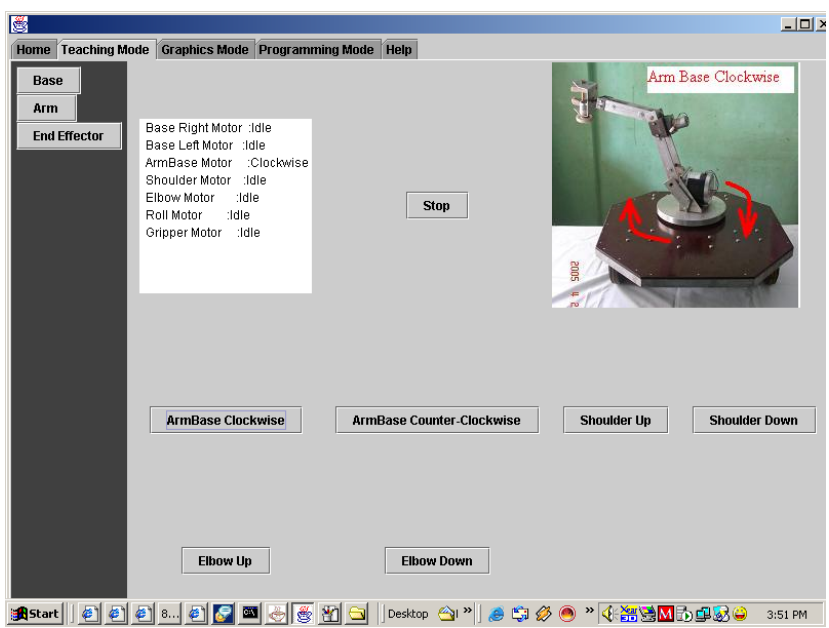

Fig. 7: GUI showing arm base motor in operation

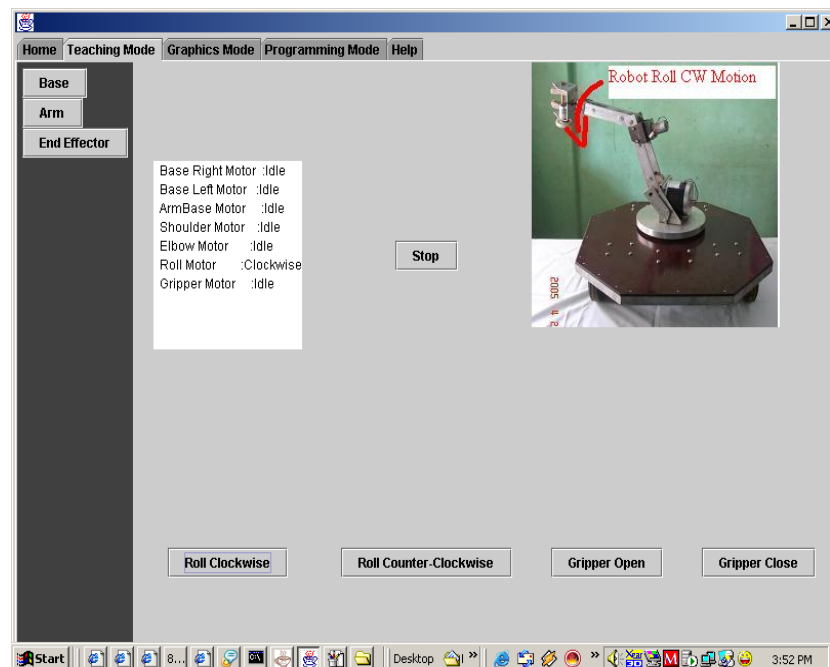

Fig. 8 : GUI showing the roll motor in operation

\subsection{THE APPLICATION GUI}

The screens used in the application is a screen, which combines all the features of the PROGRAMMING MODE, MANUAL MODE, RUN MODE and the GRAPHICAL MODE.

\subsubsection{PROGRAMMING MODE SCREEN \& THE MANUAL MODE SCREEN}

These are the default screens, loaded and displayed to the user on startup. The software provides the user with the ability to write his / her own programs using the commands provided and allow direct control of each motor employed in mobile robot, 'Octagon'. The user generates a octagon command by simply clicking on the buttons thereby avoiding time consumed in typing a program.

\section{FEATURES}

- It is the start up screen for the software application.

- Features a complete HELP file, which can be invoked from the menu provided.

- Has a menu, which enables the user to select different options.

- Simple yet powerful interface.

- Cut - copy - paste Instruction function.

- Facility to save current program.

- Facility to save current program with a different name.

- Insert function.

- Minimization of typing.

- Editing of program is possible by use of up/down buttons.

- Manual control of each motor

- Fine positioning of the base, rack, elbow and roll.

- Facility to rotate the motors clockwise and counter clockwise

- Automatic RESET function to bring the robot to the reset/home position on startup.

- Dynamic reset function to allow the user to bring the robot to the reset position at any instant.

- Auto Teach facility. 


\subsection{ABOUT THE SCREEN}

The various component modules that are incorporated into the screen are listed as follows.

\subsubsection{MOTOR SELECTION LIST BOX}

The screen features a list box, where the user selects the desired motor. The user selects the motor by double-clicking on the motor name in the list box.

\subsubsection{MOTOR SELECTED TEXT BOX}

The selected motor is then highlighted and displayed in the motor selected textbox.

\subsubsection{DIRECTION OPTION BUTTONS}

The clockwise option button is enabled by default.

The user can change the direction of rotation prior to pressing the start button. The user is required to click on the option button for selection of the direction.

\subsubsection{START BUTTON}

The START button, when clicked, enables rotation of the desired motor. On clicking the start button, all the remaining components on the screen except the stop button are disabled as a precautionary measure.

\subsubsection{STOP BUTTON}

The STOP button, when clicked, disables rotation of the desired motor by sending byte 0 to the port. On clicking the stop button, all the components disabled by the start button are re-enabled.

\subsubsection{STATUS SIGNALS and MESSAGES}

The status signals and messages are an indication, to the user, of the motor selected, the direction of rotation and the byte sent to the port.

\subsubsection{RESET BUTTON}

The screen features a reset button that is coded to respond to the click event. On clicking the button the software brings the robot to the HOME position or reset position. The reset is an important function, which is used in conjunction with the auto-teach facility provided in the manual mode screen since it is mandatory that any teaching process be preceded by a reset, so that all calculations made by the robot software are with respect to the reset position of the robot [6].

\subsection{AUTO TEACH FACILITY}

The manual mode provides the user with an auto teach facility, in which the program calculates the time delays and the associated angles, corresponding to the sequence of manually activated motors in an operation.

Semi-automatic mode teach facility is explained in this section in Fig. 10. This is the programmed mode of operation of the robot. This mode of operation is used when the user wants to perform tasks that are of repetitive nature. This mode can also be used as a static repository of all the actions that the user makes the robot perform on the Manual Mode.
Table 1: Different sub-routine modules used to maneuver the octagon using the GUI

\begin{tabular}{|l|l|}
\hline \multicolumn{1}{|c|}{$\begin{array}{c}\text { Mobile } \\
\text { (8 motions) }\end{array}$} & \multicolumn{1}{c|}{$\begin{array}{c}\text { Arm } \\
\text { (10 motions) }\end{array}$} \\
\hline Forward & Arm base clockwise \\
\hline Backward & Arm base anticlockwise \\
\hline Forward Left & Arm shoulder up \\
\hline Forward Right & Arm shoulder down \\
\hline Backward Left & Arm elbow up \\
\hline Backward Right & Arm elbow down \\
\hline Spin Clockwise & Arm roll clockwise \\
\hline Spin Anti-clockwise & Arm roll counter-clockwise \\
\hline \multicolumn{2}{|l}{} \\
\hline & Arm gripper open \\
\hline
\end{tabular}

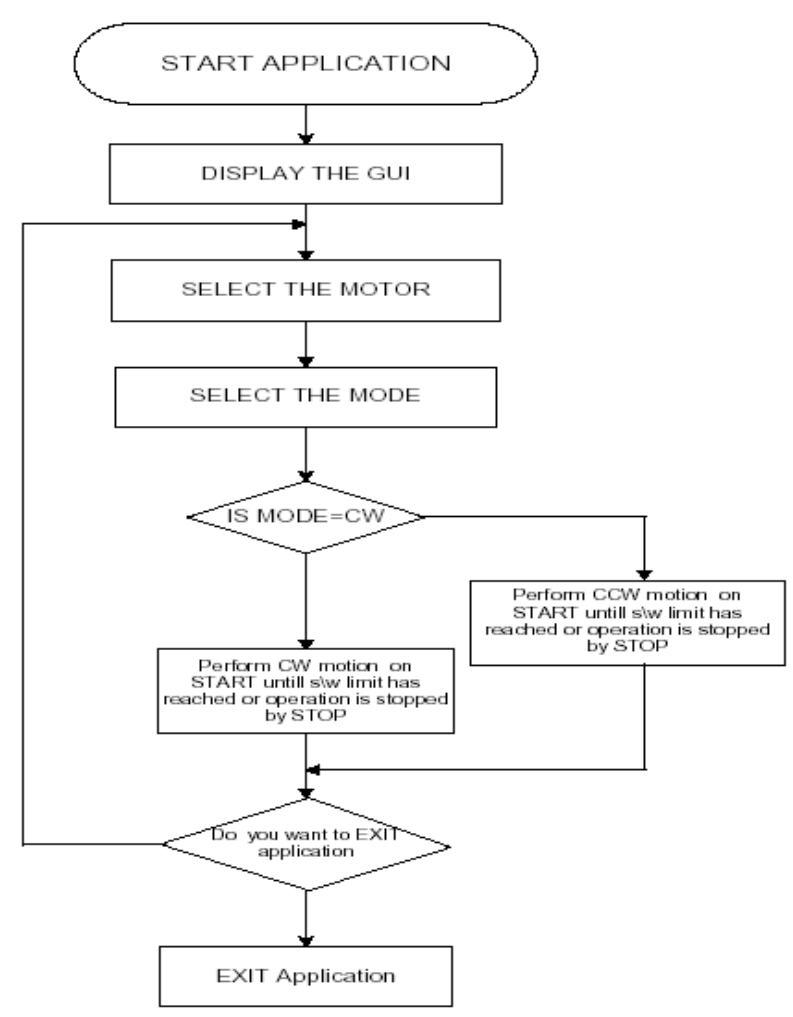

Fig. 9 : Flow-chart module of software module

In this mode of operation, the user writes a program for the operations of the robot using simple commands like moving, looping, looping end and then running the program to perform the intended operations with the robot. The user may create as many programs for the robot and save them for later use as well and load and run earlier saved programs as and when required. Looping commands allow the users to perform a set of commands again and again and this mode allows 10 level of nesting possible. 


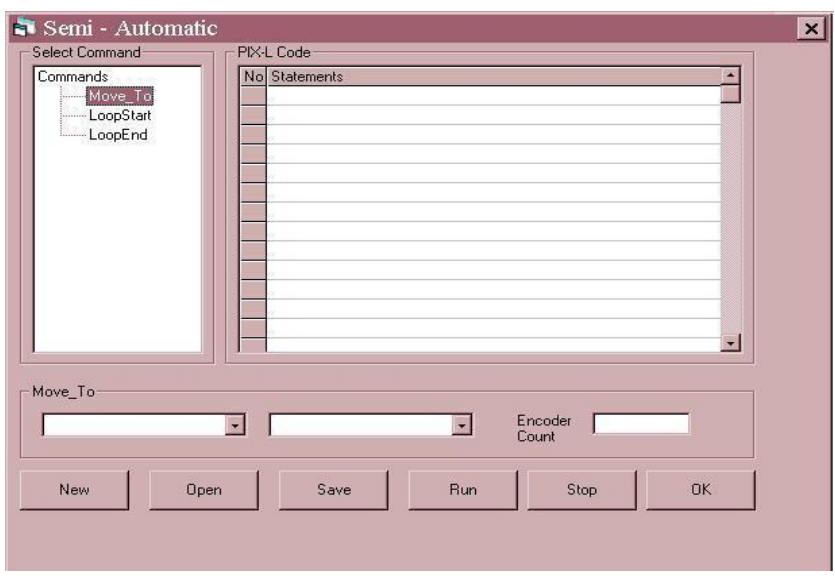

Fig. 10 : Semi-automatic teach facility

The semi-automatic mode internally depends on the manual mode for its operation and effectively translates the semi-automatic commands in a way that is understood by the manual mode [7]. The user can track all the individual actions that he performs in the manual mode. This is transferred to the semi-automatic mode in the form of a program. The user can then save this history as a program \& can repeat this whenever he desires, by simply loading $\&$ executing the program.

\subsection{SOFTWARE FOR OCTAGON MANOEURING}

The application facilitates user interaction with OCTAGON has the following features

- REAL TIME MANUAL MODE CONTROL.

- REAL TIME RUN MODE CONTROL.

- PROGRAMMING INTERFACE.

- FACILITY TO LOOP OPERATIONS.

- aCCURATE POSITIONING.

- PARALLEL PORT INTERFACE \& CONTROL.

- INTELLIGENT PATH DECISION MAKING.

\subsubsection{Communication with the octagon}

The communication between the octagon and the computer is through the PC parallel port using the data lines, status lines and the control lines as shown in the Fig. 11. To conclude as to why JAVA is used for our programming purposes is as follows. JAVA is a simple, object-oriented, network-savvy, interpreted, robust, secure, architecture neutral, portable, high-performance, multithreaded, dynamic language, which provides you with a complete set of tools to simplify rapid application development. We wanted to use a system that could be programmed easily without a lot of esoteric training and which leveraged today's standard practice. JAVA is object-oriented design and is also the mechanism for defining how modules 'plug and play'.

Java has an extensive library of routines for coping easily with TCP/IP protocols like HTTP and FTP. This makes creating network connections much easier than in $\mathrm{C}$ or $\mathrm{C}++$ and also the machine can be controlled from a remote location. Java applications can open and access objects across the net via URL's with the same ease that programmers are used to when accessing a local file system and is network savvy. The JAVA code designed runs to around. Some of the codings that was used in our GUI design for controlling the robot are shown below.

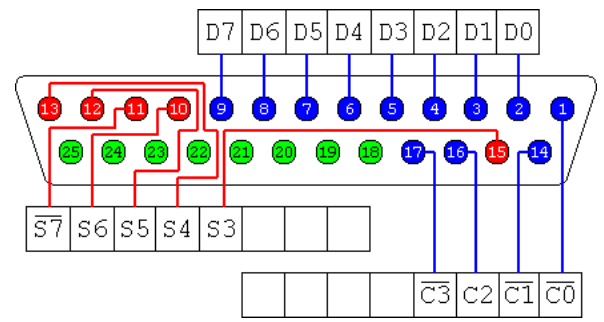

Fig. 11: 25-way Female D-Type connector

Parallel Port Interface Using JNI public class ParallelPort \{

/** The port base address (e.g. 0x378 is base address for LPT1) $* /$

private int portBase;

/** To cunstruct a ParallelPort object,

* you need the port base address */

public ParallelPort (int portBase)

\{

this.portBase $=$ portBase;

\}

/** Reads one byte from the STATUS pins of the parallel port.

* The byte read contains 5 valid bits, corresponing to 5 pins of input

* from the STATUS pins of the parallel port (the STATUS is located

* at "portBase $+1 "$ ", e.g. the STATUS address for LPT1 is 0x379).

* This diagram shows the content of the byte:

* Bit | Pin \# | Printer Status | Inverted

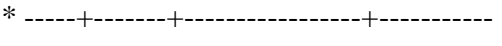

* 7 | 11 | Busy | Yes

* 6 | $10 \mid$ Acknowledge

* 5 | 12 | Out of paper |

* 4 | 13 | Selected |

* 3 | 15 | I/O error

* Note that Pin 11 is inverted, this means that "Hi" input on pin

* means 0 on bit 7, "Low" input on pin means 1 on bit 7. */

public int read ()

\{

return ParallelPort.readOneByte (this.portBase+1);

\}

/** Writes one byte to the DATA pins of parallel port.

* The byte is written to the DATA pins of the port. The DATA pins are

* located at the base address of the port (e.g. DATA address for LPT1

* is $0 \times 378)$.

* This diagram shows how the byte is written: 


\begin{tabular}{cc|c|c}
$*$ & Bit & Pin \# & Printer DATA \\
$*$ & ---+------- \\
$*$ & 7 & 9 & DATA 7 \\
$*$ & 6 & 8 & DATA 6 \\
$*$ & 5 & 7 & DATA 5 \\
$*$ & 4 & 6 & DATA 4 \\
$*$ & 3 & 5 & DATA 3 \\
$*$ & 2 & 4 & DATA 2 \\
$*$ & 1 & 3 & DATA 1 \\
$*$ & 0 & 2 & DATA 0 \\
$*$ & & &
\end{tabular}

public void write (int oneByte)

\{

ParallelPort.writeOneByte(this.portBase, oneByte);

\}

I** Reads one byte from the specified address.

* (normally the address is the STATUS pins of the port) */

public static native int readOneByte (int address);

/** Writes one byte to the specified address

* (normally the address is the DATA pins of the port) */

public static native void writeOneByte (int address, int oneByte);

static

\{ System.loadLibrary("parport"); \}

\}

We have used 2 methods to interact with ParallelPort :

\section{1) read}

public int read()

Read one byte from the STATUS address of a parallel port. The byte read contains 5 valid bits, corresponding to 5 pins of input from the STATUS pins of the parallel port (the STATUS is located at "portBase + 1", e.g. the STATUS address for LPT1 is 0x379).

\section{2) write}

public void write(int oneByte)

Write one byte to the DATA address of a parallel port. The byte is written to the DATA pins of the port. The DATA pins are located at the base address of the port (e.g. DATA address for LPT1 is 0x378).

\section{CONCLUSIONS}

An indigenously developed mobile robotic system with the help of a user friendly GUI is presented in this paper. The mechanical assembly was divided into 3 units, viz., base, arm and the endeffector assembly. The electronic section was done in 3 stages, viz., power supply, controller and the driver unit. The robot was controlled using a GUI developed in JAVA in various modes. A number of pick and place operations were successfully performed by the developed robot using various types of modes.

\section{REFERENCES}

[1] Patrick Naughton, Michael Morrison, "JAVA Handbook", Osborne / McGraw-Hill Publishes, 1996.

[2] Doug Lea, "Concurrent Programming in Java: Design Principles and Patterns", Addison-Wesley, 1996.

[3] Argyros A., Geordiadis P., Trahanias P., and Tsakiris D., 'Semiautonomous navigation of a robotic wheelchair', Intelligent and Robotic Systems, 34, 315-329, (2002).

[4] Lankenau A. and Röfer T.A., 'A versatile and safe mobility assistant', Robotics and Automation Magazine, 8(1), (March 2001).

[5] Kuipers B.J., 'The spatial semantic hierarchy, in artificial intelligence', Artificial Intelligence, 119, 191-233, (2000).

[6] Galindo C., Fernandez J.A., and Gonzalez J., 'Improving efficiency in mobile robot task planning through world abstraction', IEEE Transaction on Robotics and Automation.

[7] Fernandez J.A. and Gonzalez J., Multi-Hierarchical Representation of Large-Scale Space, Kluwer Academic Publishers, 2001.

[8] Fernandez J.A. and Gonzalez J., 'Multihierarchical graph search', IEEE Transaction on Pattern Analysis and Matching, 24(1), (2002).

[9] Hirtle S.C. and Jonides J., 'Evidence of hierarchies in cognitive maps', Memory and Cognition, 13(3), 208-217, (1985).

[10] Yuchul Jung, Yong K. Hwang, Manjai Lee, "Case-Based Reasoning Approach toTask Planning of Home-Service Robots", ICAT 2004.

[11] Roman Zahariev, Dimitar Karastoyanov, “A Navigation System and Task Planning in a Mobile Robot for Inspection", Bulgarian academy of sciences, Conference paper, 2004.

[12] Robert, J.S., Fundamentals of Robotics : Analysis and Control, PHI, New Delhi., 1992.

[13] Klafter, Thomas and Negin, Robotic Engineering, PHI, New Delhi, 1990.

[14] Fu, Gonzalez and Lee, Robotics : Control, Sensing, Vision and Intelligence, McGraw Hill, Singapore, 1995.

[15] Ranky, P. G., C. Y. Ho, Robot Modeling, Control \& Applications, IFS Publishers, Springer, UK., 1998.

[16] T.C.Manjunath, Fundamentals of Robotics, Nandu Publishers, $5^{\text {th }}$ Revised Edition, Mumbai., 2005.

[17] T.C.Manjunath, Fast Track To Robotics, Nandu Publishers, $3^{\text {nd }}$ Edition, Mumbai, 2005.

[18] Ranky, P. G., C. Y. Ho, Robot Modeling, Control \& Applications, IFS Publishers, Springer, UK, 2005.

[19] Groover, Weiss, Nagel and Odrey, Industrial Robotics, McGraw Hill, Singapore, 2000.

[20] William Burns and Janet Evans, Practical Robotics Systems, Interfacing, Applications, Reston Publishing Co., 2000.

[21] Phillip Coiffette, Robotics Series, Volume I to VIII, Kogan Page, London, UK, 1995. 
[22] Luh, C.S.G., M.W. Walker, and R.P.C. Paul, On-line computational scheme for mechanical manipulators, Journal of Dynamic Systems, Measurement \& Control, Vol. 102, pp. 69-76, 1998.

[23] Mohsen Shahinpoor, A Robotic Engineering Text Book, Harper and Row Publishers, UK.

[24] Janakiraman, Robotics and Image Processing, Tata McGraw Hill.

[25] Richard A Paul, Robotic Manipulators, MIT press, Cambridge.

[26] Robin JAULMES, Joelle PINEAU and Doina PRECUP, “A formal framework for robot learning and control under model uncertainty", IEEE International Conference on Robotics and Automation (ICRA), 2007.

[27] Fairhunt, "Computer Vision for Robotic Systems", CFS Pubs., New Delhi.

[28] Yoram Koren, "Robotics for Engineer", McGraw Hill.

[29] Bernard Hodges, "Industrial Robotics", Jaico Publishing House, India.

[30] Tsuneo Yoshikawa, "Foundations of Robotics : Analysis and Control", $P H I$, India.

[31] Dr. Jain and Dr. Aggarwal, "Robotics : Principles \& Practice", Khanna Publishers, Delhi.

[32] Lorenzo and Siciliano, "Modeling and Control of Robotic Manipulators", McGraw Hill.

[33] Dr. Amitabha Bhattacharya, "Mechanotronics of Robotics Systems", Kaizen Publishing, Calculatta, India.

[34] S.R. Deb, "Industrial Robotics", Tata MacGraw Hill.

[35] Edward Kafrissen and Mark Stephans, "Industrial Robots and Robotics", Prentice Hall Inc. , Virginia, USA.

[36] Rex Miller, "Fundamentals of Industrial Robots and Robotics", PWS Kent Pub Co., Boston, USA.

[37] Doughlas R Malcom Jr., "Robotics ... An introduction", Breton Publishing Co., Boston .

[38] Wesseley E Synder, "Industrial Robots : Computer Interfacing and Control", Prentice Hall.

[39] Carl D Crane and Joseph Duffy, "Kinematic Analysis of Robot Manipulators", Cambridge Press, UK.

[40] CY Ho and Jen Sriwattamathamma, "Robotic Kinematics ... Symbolic Automatic and Numeric Synthesis", Alex Publishing Corp, New Jersey.

[41] Deepali Trivedi, Amit Joshi, SA Patekar, "Design \& development of mobile robot-octagon", M.Tech. Project Report, VJTI, Matunga, 2006.

\section{AUTHOR PROFILE}

Dr. T.C.Manjunath was born in Bangalore, Karnataka, India on Feb. 6, 1966 \& received the B.E. Degree (Bachelor of Engg.) in Electrical Engg. from R.V. College of Engg. (Bangalore University) with M.Tech. degree in Electrical Engg. with specialization in Automation, Control \& Robotics from L.D. College of Engg. (Gujarat University) and Ph.D. from the prestigious Indian Institute of Technology Bombay (IIT Bombay) in Systems \& Control Engg., respectively. I have got a teaching experience of nearly 22 long years in various engineering colleges all over the country (Karnataka, Tamilnadu, Gujarat, Maharashtra) as Lecturer, Asst. Prof., Professor, Head of the Department, Principal \& as Director. . I also worked as a Project Assistant and as a Research Engineer in the Systems and Control Engineering (IIT Bombay, India) and worked on control of space launch vehicles using FOS feedback technique in IITB. I have published a number of publications in the various National, International journals and Conferences and published 3 textbooks on Robotics, one of which has gone upto the fourth edition, titled, 'Fast Track to Robotics' and the other, which has gone upto the fifth edition, titled, 'Fundamentals of Robotics' in 2 volumes, Vol.-1 and Vol.2 along with a CD which contains about $150 \mathrm{C} / \mathrm{C}++$ programs for performing various simulations on robotics. I have also published a research monograph cum patent in the International level from the Springer-Verlag publishers based on his Ph.D. thesis topic titled, "Modeling, Control and Implementation of Smart Structures", Vol. 350, LNCIS, costing 79.95 Euros. I am a member of IEEE for the past 9 years, SPIE student member and IOP student member for 4 years, life member of ISSS (India), life member of the ISTE (India), life member of ISOI (India), life member of SSI (India) and life member of the CSI (India) and life member cum fellow of the IETE (India). I have also presented a number of guest lectures and seminars in many institutions across the country and participated in more than 2 dozen CEP / DEP courses, seminars, workshops, symposiums in the various parts of the country in different institutions and also conducted a few courses in the institutions where I have worked. I was awarded with the "Best research scholar award in engineering discipline" for the academic year 2006-07 from the Research Scholars Forum (RSF) from Indian Institute of Technology Bombay (IITB). This award was presented in recognition of the significant contribution to the research (amongst all the researchers in all disciplines) in IIT Bombay. I was also instrumental in getting Research centres along with M.Tech programs in the colleges where I have worked so far. I was also responsible for getting a AICTE grant of Rs. 10 Lakhs under MODROB scheme for the development of the Robotics \& Mechatronics Lab in Fr. CRCE, Mumbai. Apart from which, I have brought couple of grants for the conduction of various events like workshops \& conferences where I have worked. I have visited Singapore, Russia, United States of America and Australia for the presentation of his research papers in various international conferences. My biography was published in $23^{\text {rd }}$ edition of Marquis's Who's Who in the World in the 2006 issue. I have also guided more than 2 dozen projects (B.Tech. I M.Tech.) in various engineering colleges where I have worked so far apart from guiding a couple of research scholars who are doing $\mathrm{Ph} . \mathrm{D}$. in various universities. Many of my guided projects, interviews have appeared in various national newspapers and magazines. I have also reviewed many research papers for the various international conferences such as IEEE IECON-06, IEEEISIE-2007, NSC-07, IEEE-WCSN-07, etc., and has also reviewed many journal papers for journals such as IJAMT, etc.,. I am also a editorial board member on many of the international Journals like IJRIC, IJCEE (Singapore), IJCTE (Singapore), IJET (Singapore), IST Press (4 journals). I have also given many invited talks / plenary lecturers in various national \& international conferences and chaired many sessions. 